\title{
Effect of Prolonged Sitting and Breaks in Sitting Time on Endothelial Function
}

\author{
Saurabh S. Thosar ${ }^{1}$, Sylvanna L. Bielko ${ }^{1}$, Kieren J. Mather ${ }^{2}$, \\ Jeanne D. Johnston ${ }^{1}$, and Janet P. Wallace ${ }^{1}$ \\ ${ }^{1}$ Department of Kinesiology, Indiana University School of Public Health, Indiana \\ University Bloomington, IN $;^{2}$ Department of Medicine, Indiana University School of \\ Medicine, Indianapolis, IN
}

\section{Corresponding author:}

Saurabh S. Thosar, PhD.

Postdoctoral Researcher,

Oregon Institute of Occupational Health Sciences,

Oregon Health and Science University, L606/RJH1553A,

Portland, Oregon 97239.

Phone: (503)-494-2064; Fax: (503)-494-3849

Email: thosar@ohsu.edu

Running title: Prolonged sitting and breaking sitting time

Funding sources: This project was funded in part through American College of Sports Medicine

Foundation's doctoral research grant, Indiana University Graduate School's grant-in-aid and Indiana University School of Public Health's grant-in-aid.

Conflict of interest: The authors declare no conflict of interest

This is the author's manuscript of the article published in final edited form as:

Thosar, S. S., Bielko, S. L., Mather, K. J., Johnston, J. D., \& Wallace, J. P. (2015). Effect of prolonged sitting and breaks in sitting time on endothelial function. Medicine and Science in Sports and Exercise, 47(4), 843-849. http://doi.org/10.1249/MSS.0000000000000479 


\section{Abstract}

Sitting time (ST) is associated with cardiovascular disease risk factors whereas breaking ST has been reported to be beneficial for reducing cardiovascular risk. PURPOSE: To examine the effects of breaking ST on superficial femoral artery (SFA) endothelial function. Hypotheses: 1) prolonged sitting would induce endothelial dysfunction and changes in shear forces and 2) breaking sitting time with brief periods of activity would prevent attenuation in endothelial function. METHODS: Twelve non-obese men (24.2 \pm 4.2 yrs.) participated in two randomized 3hr sitting trials. In the sitting (SIT) trial, subjects were seated on a firmly cushioned chair for $3 \mathrm{hr}$ without moving their lower extremities. In the breaking sitting time trial (ACT), subjects sat similar to the SIT trial, but walked on a treadmill for five min at $2 \mathrm{miles} \cdot \mathrm{hr}^{-1}$ at $30 \mathrm{~min}, 1 \mathrm{hr}$ 30min, and $2 \mathrm{hr} 30 \mathrm{~min}$ during the sitting interval. SFA flow mediated dilation (FMD) was assessed at baseline, $1 \mathrm{hr}, 2 \mathrm{hr}$, and $3 \mathrm{hr}$ in each trial. Statistical analyses were performed using dependent variables SFA FMD, and shear rates. Significance was set at $p \leq 0.05$. RESULTS: In the SIT trial, there was a significant decline in SFA FMD from baseline to $3 \mathrm{hr}$ (baseline: 4.72 $\pm 3.78 \%$, 1hr: $0.52 \pm 0.85 \%, 2 \mathrm{hr}: 1.66 \pm 1.11 \%, 3 \mathrm{hr}: 2.2 \pm 2.15 ; \mathrm{p}<0.05$ by ANOVA) accompanied by a decline in mean shear rate (MSR) and antegrade shear rate (ASR), but no difference in SRauc. By 2-way repeated measures ANOVA, ACT prevented the sitting-induced decline in FMD, (baseline: 4.5 $\pm 2.3 \%, 1 \mathrm{hr}: 5.04 \pm 2.85 \%, 2 \mathrm{hr}: 5.28 \pm 5.05 \%, 3 \mathrm{hr}: 6.9 \pm 4.5 \%$ ) along with no decline in shear rates. CONCLUSION: Three hours of sitting resulted in a significant impairment in shear rate and SFA FMD. When light activity breaks were introduced hourly during sitting, the decline in FMD was prevented.

Keywords: Sedentary activity, flow mediated dilation, intermittent activity, low shear rate 
Introduction

Prolonged sitting is a common (8) but deleterious (1) behavior in contemporary humans. Increased sitting time has been associated with chronic diseases and increased risk of mortality even while controlling for leisure time physical activity(1). Epidemiological evidence also suggests that sitting time is directly associated with the biomarkers of cardiovascular diseases (CVD) such as total cholesterol, LDL cholesterol, triglycerides, waist circumference and blood pressure $(6,25)$. In contrast, breaking sitting time has been associated with beneficial patterns of CVD risk markers including lower waist circumference, triglycerides, and $2 \mathrm{hr}$ plasma glucose(9). In the limited literature examining the beneficial physiological effects of breaking sitting time, Dunstan et al(4) reported that breaking sitting time via low intensity and moderate intensity activity attenuated the rise in postprandial glucose and insulin that were observed after a test meal in an acute bout of $7 \mathrm{hr}$ of sitting. Similarly, Stephens and colleagues (32) observed a $39 \%$ reduction in insulin action in a day long sitting condition as compared to the active condition. Further, Duvivier et al (5) found breaking sitting time was beneficial for plasma lipids and insulin sensitivity even more than adding one hour of vigorous intensity physical exercise for four days.

In the past three decades impaired endothelial function has emerged as a predictor of CVD risk(3), and in some studies is shown to be at least as predictive as traditional risk factors(37)In fact, endothelial function is impaired even before the traditional risk factors are expressed (7). The effects of prolonged sitting on the endothelial function, and the potential beneficial effects of breaking sitting time, have not been previously published. We have proposed that prolonged sitting may lead to a shear-mediated endothelial dysfunction (35). The purpose of this study was to investigate the effects of $3 \mathrm{hrs}$ of sitting on lower extremity mean 
shear rate and endothelial function, and to study whether breaking sitting time modulated these effects. We hypothesized that $3 \mathrm{hrs}$ of sitting would lead to a decline in mean shear rate, and decline in superficial femoral artery (SFA) endothelial function as measured by flow mediated dilation (FMD). A second hypothesis was that systematic low intensity physical activity (5 min at 2 miles $^{-1} r^{-1}$ each hour) used to break this sitting time would mitigate the decline in endothelial function.

Methods

Study design (Figure 1): This study consisted of two screening visits and two sitting trials in random sequence, one with uninterrupted sitting (SIT) and one with breaks in sitting time (ACT). FMD measurements were conducted at baseline, $1 \mathrm{hr}, 2 \mathrm{hr}$ and $3 \mathrm{hr}$ in both the trials. All procedures for the study were approved by Indiana University Institutional Review Board, and participants gave written informed consent for their participation.

Subjects: To be included, subjects had to self-report that they were nonsmokers, and not taking any anti-hypertensive, lipid lowering, and/or anti-diabetic medications. To be included, they needed to have total cholesterol $\leq 240 \mathrm{mg} \cdot \mathrm{dl}^{-1}$ and triglycerides $\leq 200 \mathrm{mg}^{-\mathrm{dl}^{-1}}$. We recruited individuals who performed $<150$ min.week $^{-1}$ of moderate intensity physical activity or $<75$ min week $^{-1}$ of vigorous intensity physical activity. We intentionally chose a group of subjects who do not meet the physical activity guidelines recommended by the Centers for Disease Control and Prevention (2). Subjects were asked to maintain their regular diet patterns throughout the study duration and to discontinue any over the counter antioxidant supplement at least 7 days prior to the first session. Based on our pilot data, for the primary dependent variable SFA FMD, we estimated that 12 subjects would be needed to find a significant difference between trials with statistical power of $>0.80$ at $\alpha \leq 0.05$. 
Screening visits: At the first visit, all experimental procedures were explained to the subjects and they were familiarized with the lab setting. After obtaining a written informed consent, height, weight and blood pressure were measured using standard procedures and a medical health history and habits questionnaire (12) was completed to screen for any preexisting medical conditions and to physical activity. Blood pressure was measured during an additional screening visit, at the same time of day, to confirm previous measurements.

Testing trials

Subjects arrived at the laboratory after an overnight fast of at least 6 hours between 0700 and 0900 hours. The arrival time was matched for both trials within each subject. Subjects were also asked to refrain from any caffeinated drink for at least 8 hours before reporting to the lab. Upon their arrival in the lab, all subjects self- reported adhering to the fasting schedule. During both trials, the subjects remained seated for $3 \mathrm{hrs}$ without moving their legs (perpendicular to the ground) or feet (resting on the floor). Subjects were seated for $\sim 5-10 \mathrm{~min}$ before the first measurement. Subjects were allowed to move their arms, not vigorously, for example to use a computer or do light reading during the non-testing periods of the trials. Arm movement was not quantified. The FMD and other vascular function parameters were measured at baseline, $1 \mathrm{hr}, 2 \mathrm{hr}$ and $3 \mathrm{hr}$. The two trials were performed in random order, separated by a minimum of two and a maximum of seven days. The SIT trial was uninterrupted sitting for $3 \mathrm{hrs}$ whereas in the ACT trial subjects completed three, 5 minute bouts of walking at 2 miles $\mathrm{hr}^{-1}$ on an adjacent treadmill at $30 \mathrm{~min}, 1 \mathrm{hr} 30 \mathrm{~min}$ and $2 \mathrm{hr} 30 \mathrm{~min}$ during the sitting interval. 
Superficial femoral artery FMD

Endothelial function was measured using SFA FMD was measured in accordance with current guidelines (34). We chose SFA for two reasons: (1) It is a readily accessible artery to for measuring FMD in a sitting position; and (2) second, SFA FMD has been shown to be largely nitric oxide (NO) dependent (15). Each measurement was performed in a dark, quiet and climate controlled room $\left(22-25^{\circ} \mathrm{C}\right)$ room. A $5 \times 84 \mathrm{~cm}$ automatic blood pressure cuff $(\mathrm{E}-20$ rapid cuff inflator; D.E. Hokanson, Bellevue, Wash., USA) was placed on their right thigh about $7 \mathrm{~cm}$ above the knee joint, distal to the SFA recording location. Images of the SFA were obtained longitudinally $7-10 \mathrm{~cm}$ below the inguinal line with a 2-D high-resolution ultrasound system (Terason t3000, Teratech Corp., Burlington, Mass., USA), using a 5- to 12-MHz multifrequency linear-array transducer. Once satisfactory images of near and far arterial walls were obtained, the transducer was secured and stabilized in a stereotactic clamp, and landmarks were made on the subject's skin to ensure similar placement of the transducer for subsequent FMD procedures and shear rate assessments within and between conditions. Subjects were encouraged to keep the landmarks between the 2 trials. In addition to imaging the arterial dimensions, Doppler ultrasound was used to concurrently measure SFA blood velocity. Doppler flow signals were corrected at an insonation angle of $60^{\circ}$, and the sample volume was placed in the middle of the artery.

Diameter images and Doppler measurements of blood velocity were continuously recorded for $45 \mathrm{~s}$ at baseline prior to cuff inflation. The automatic blood pressure cuff was then rapidly inflated to $250 \mathrm{mmHg}$ and maintained for $5 \mathrm{~min}$ until cuff deflation. Diameter and blood velocity recordings resumed prior to cuff deflation and continued for 5 min after deflation. Ultrasound images were continuously recorded at 5 frames $\cdot \mathrm{s}^{-1}$ with Camtasia (TechSmith, 
Okemos, Mich., USA), and stored as .avi files $(12,21)$. This procedure was repeated hourly across the sitting intervals.

Arterial diameters and blood velocities: Off-line analysis of diameters were performed using automated edge-detection software (Brachial Analyzer, Medical Imaging Applications LLC, Coralville, IA, USA) as previously described(21). This software allows the technician to determine a region of interest where the near and far vessel walls are most clear. The vessel wall borders are then detected using an optimal graph search-based segmentation that uses a combination of pixel density and image gradient as an objective function. All analyzed images were reviewed by the technician and edited when needed to ensure that diameter measures were always determined from the intima-lumen interface at the near and far vessel wall. Blood velocities were determined using custom made software that we developed for this project by selecting a region of interest that surrounded the Doppler wave. The velocity-time integral was used to calculate the mean blood velocity for each cardiac cycle. Diameters and blood velocities were not ECG-gated $(14,26)$. The peak dilation after cuff deflation was determined using the highest 3-s moving average and was presented as a percentage change from baseline diameter (FMD\%). SFA shear rate used as an estimate of arterial shear stress and was calculated for each FMD $\%$ at baseline and during the post occlusion period using the following formula: $4 \cdot \mathrm{Vm} \cdot \mathrm{D}^{-1}$, where $\mathrm{V}$ m is mean blood velocity $\left(\mathrm{cm} \cdot \mathrm{s}^{-1}\right)$ and $\mathrm{D}$ is mean arterial diameter $(\mathrm{cm})$. All measurements and analysis were performed by a single researcher (ST) who was blinded to the participant identity and treatment condition for each image file. 
Statistical analysis

Descriptive analysis was performed to summarize subject characteristics. Within both trials, one way ANOVA was conducted on the baseline diameter as the dependent variable. Within the SIT trial, a one way ANOVA was conducted on the dependent variables baseline diameter, FMD\%, and shear rates (antegrade shear rate, mean shear rate, retrograde shear rate, peak shear rate and shear rate [area under the curve] SRauc). When an effect was found, pairwise comparisons were used to locate significant differences across time. Observed effect size was reported for ANOVA interactions and main effects as partial eta squared $\left(\eta^{2}\right)$. Comparisons across the two treatment conditions for FMD were performed using a 2-way repeated measures ANOVA, evaluating whether the effect of sitting differed between conditions; again, pairwise comparisons were used to identify significant differences at each time point if the main effects were positive. All values are expressed as the mean \pm standard error of the mean and the alpha level for statistical significance was set a priori at 0.05. All statistical calculations were performed using IBM SPSS Statistics 21.0 software (IBM SPSS Inc.).

Results

We recruited and tested 12 subjects, who comprised a homogenous group of apparently healthy inactive young men (Table 1). We were unable to collect data for one time point FMD on one subject in the ACT trial and this subject's data was excluded from the two way ANOVA between trials.

SIT trial: Superficial femoral artery baseline diameters are presented in Table 2. There was no significant difference between the baseline diameters from baseline to $3 \mathrm{hr}\left(\eta^{2}=0.112, \mathrm{p}=\right.$ 0.263). 
Superficial femoral artery FMD (Figure 2): There was a significant reduction in FMD across time in SIT each hour, from baseline to $3 \mathrm{hr}\left(\eta^{2}=0.481, \mathrm{p} \leq 0.001\right)$. The FMD was significantly reduced at $1 \mathrm{hr}, 2 \mathrm{hr}$ and $3 \mathrm{hr}$ as compared to baseline. There was no significant difference from baseline to $3 \mathrm{hr}$ in retrograde shear rate, peak shear rate and SRauc ( $>>0.05)$. Baseline antegrade shear rate: A significant decline in the antegrade shear rate from baseline to $3 \mathrm{hr}\left(\eta^{2}=0.209, \mathrm{p}=\right.$ 0.049). The antegrade shear rate was significantly lower at $1 \mathrm{hr}$ and $2 \mathrm{hr}$ as compared to baseline. Mean shear rate: There was a significant decline in mean shear rate from baseline across time $\left(\eta^{2}\right.$ $=0.237, \mathrm{p}=0.028)$. Mean shear rate was significantly lower at $1 \mathrm{hr}$ and $2 \mathrm{hr}$ as compared to baseline. Shear rate data are presented in Table 3.

SIT and ACT trial: A $2 \times 4$ (trial $\times$ time) repeated measures ANOVA was performed for the dependent variable FMD in the SIT and ACT trial. There was a significant trial $\mathrm{x}$ time interaction $(\mathrm{p}<0.05)$ between FMD and time across trials. The variable of interest was the FMD difference between trials at each measurement (baseline, $1 \mathrm{hr}, 2 \mathrm{hr}$ and $3 \mathrm{hr}$ ). FMD between the two trials was not significantly different at baseline. However, FMD was significantly reduced in the SIT trial, as compared to the ACT trial at $1 \mathrm{hr}, 2 \mathrm{hr}$ and $3 \mathrm{hr}$ (figure 2).

\section{Discussion}

The purpose of this study was to examine the effects of prolonged sitting and breaking sitting time on superficial femoral artery endothelial function (flow mediated dilation). We hypothesized that $3 \mathrm{hrs}$ of sitting would lead to a decline in mean shear rate and a decline in superficial femoral artery (SFA) endothelial function as measured by flow mediated dilation (FMD). Our second hypothesis was that systematic low intensity physical activity (5 min at 2 miles $\mathrm{hr}^{-1}$ ) would prevent the decline in FMD. Indeed, we discovered a decline in mean shear rate during 3 hours of sitting. We also discovered a significant attenuation of SFA FMD on $3 \mathrm{hr}$ of 
sitting. We also observed that when breaks with modest activity were added during the sitting time, the decline in FMD was not observed. This is the first study to our knowledge to examine the direct effects of prolonged sitting and breaking sitting time on endothelial function. There are numerous sitting opportunities in today's society, including but not limited to, sitting at work, sitting during transportation, and this area is of major public health concern. Our observations are the first experimental evidence of the effects of prolonged sitting on human vasculature, and are important from a public health perspective.

Very little data has been published evaluating the effects of prolonged sitting on FMD on lower extremity vascular function. Padilla and colleagues (22) used a similar protocol to study the effects of hydrostatic load on popliteal artery FMD during $3 \mathrm{hr}$ of sitting, and reported no significant difference in the FMD in the popliteal artery after $3 \mathrm{hr}$ of sitting. Our FMD results contradict the primary findings from this study. An important difference in study design may explain these contradictory results: Padilla moved the subjects from the sitting position to the prone position to measure the endothelial function, thereby breaking the static circumstance in the leg. This active postural change may have compromised their ability to observed adverse effects on FMD, as even a simple change in position involves muscle contractions and changes in flow patterns (20). We maintained the sitting stimulus during FMD measurement and found a significant decline in SFA FMD.

On the other hand, our mean shear rate results are similar to those reported by Padilla and colleagues(22). We had expected that the mean baseline shear rate would decline over the $3 \mathrm{hr}$ of prolonged sitting (secondary to low blood flow in the legs (30) and would be maintained during breaking sitting time. Our mean shear rates during the $3 \mathrm{hr}$ of sitting significantly declined from baseline by hour 1 , and stayed low all through the $3 \mathrm{hrs}$ of sitting. Padilla observed a significant 
decline in mean shear rates after just 30 min of sitting which was maintained during $3 \mathrm{hr}$ of sitting. Their baseline measurement was, however, in the prone position. All our measurements were all in the seated position with the first measurement taken after $\sim 10-15$ min of acclimatization. Thus, our findings are more robust because our design involves repeated measurements of FMD in one position. Our results suggest that sitting creates a milieu of low mean shear rate and also leads to the decline in FMD. It is important to note that the hyperemic stimulus SRauc and retrograde shear did not change throughout the sitting trial. This implies that there was an impairment of endothelial function independent of any change in the hyperemic stimulus or retrograde shear. Another interesting finding from our study is the progressive decline in antegrade shear. Antegrade shear represents forward flow through the (superficial femoral) artery. Even in the absence of overt atherosclerosis, a chronic decrease in antegrade shear creates a pro-atherogenic milieu $(36,40)$. Hence, we can hypothesize that repeated bouts of prolonged sitting may contribute to vascular aging, at least in the lower extremities. To summarize our results from the SIT trial, $3 \mathrm{hr}$ of uninterrupted sitting leads to a progressive decline in antegrade and mean shear rate and corresponding impairment in endothelial function.

We had hypothesized that, breaks in sitting time (ACT trial) would prevent the decline in shear rates and FMD observed during the SIT trial. We found a significant difference in FMD between the SIT and ACT trials at $1 \mathrm{hr}, 2 \mathrm{hr}$, and $3 \mathrm{hr}$; with no significant difference observed at baseline. However, we did not find a significant difference in the mean shear rate between these trials. Our subjects performed light intensity physical activity $30 \mathrm{~min}, 1 \mathrm{hr} 30 \mathrm{~min}$ and $2 \mathrm{hr} 30 \mathrm{~min}$ during the sitting interval, and we measured flow at $1 \mathrm{hr}, 2 \mathrm{hr}$ and $3 \mathrm{hr}$. Even this very light intensity physical activity prevented the decline in FMD that was seen in the SIT trial, and may explain the protective effect against sitting-induced impairment in FMD. Our mean shear rate 
observations between trials are not different perhaps because of the timing of measurement. There was a lag of $\sim 25 \mathrm{~min}$ between the activity and the shear rate measurement. It is possible that the flow ephemerally increased on walking and had decreased closed to baseline been attenuated at the time of measurement.

Mechanisms: There are at least three possible mechanisms explaining the attenuation in FMD during sitting and it's mitigation after breaking sitting time. These mechanisms may act separately or may be integrated.

First, in the seated position, the shear rates are lower than in supine position(20). Once sitting commences, these shear rates initially decline and are maintained creating a condition of low flow in the lower extremity(22) (also seen in our measurements). Conditions of low flow have been associated with impaired endothelial function(19). In our study, we found a decline in SFA FMD during 3 hours of sitting which was associated with concurrent reductions in shear forces. Although no prior evidence exists which directly demonstrates the effects of exercise on shear in the SFA, it is logical that the low intensity activity in our study episodically increased shear rate in the SFA (and other leg vessels). The brief intervals of low-level activity in our ACT trial appear to have been sufficient to prevent sitting-related reductions in shear.

A second possible mechanism can be blood viscosity. Viscosity has been shown to be higher in the legs after $2 \mathrm{hr}$ of sitting(11). Increased viscosity has been associated with increased coagulation and inflammatory markers(18) which in turn have been strongly associated with impaired endothelial function(39). Low shear rates are associated with clumping of red blood cells and increased viscosity(17). Conversely, increased shear rates from walking (ACT) (33) would intermittently oppose the rise in viscosity and coagulation markers, resulting in preserved FMD. 
A third possibility is an increased muscle sympathetic nerve activity (MSNA). MSNA is higher during the upright sitting posture(13) and along with increased blood pressure(30) may result in a decline in FMD(10). Indeed, increased MSNA is also linked to increased pro-atherogenic shear patterns in the conduit arteries(23). Walking induced muscular contractions would help in venous return thereby not requiring the sympathetic outflow compensation during prolonged sitting(24). In addition, light intensity activity has been known to directly decrease MSNA(29). In our study, the light intensity walking may have ephemerally lowered the MSNA and preserved shear patterns and FMD.

The burden of atherosclerotic lesions in the human peripheral vasculature is greater in the lower extremities than the upper extremities $(16,28,31)$. Sitting is a common activity in today's society. Based on ur data in addition to the previous work on the adverse effects of sitting (20, 22) we can hypothesize that perhaps repeated bouts of prolonged sitting result in chronic low shear rates in the lower extremities which impair endothelial function and accelerate the atherosclerotic process in the lower extremity. Because the upper extremity is constantly mobile for activities of daily living, even during the seated position, upper extremity endothelial function may be is relatively protected from the harmful effects of sitting.

Limitations: Potential limitations of this study should be considered. We did not directly measure blood pressure or MSNA (13)in the lower extremity in the seated position. Padilla and colleagues(22) found leg blood pressure was significantly higher during $3 \mathrm{hr}$ of sitting than at baseline. Blood pressure may be one of the mechanisms contributing to the decline in $\operatorname{FMD}(27)$. We considered measuring blood pressure; however the occlusion of the cuff may have resulted in changes in the shear rates. The seated posture is known to increase MSNA (13) .However, MSNA measurement would have compromised the strict non-movement of the legs, and thereby 
our study design. We did not measure viscosity. Since viscosity is an integral component of shear stress, viscosity measurement would have provided us with actual shear stress results without using shear rate as a surrogate measure for shear stress. However, viscosity measurement would have been relevant only if the blood was sampled from the legs. We attempted repeated blood draws from the lower extremities but were unsuccessful. Finally, absence of blood biomarkers sampled from the lower extremity is a clear limitation as we cannot relate locally sampled factors to low shear related mechanism and declining SFA FMD during sitting. As noted earlier, before the beginning of data collection we attempted to master the technique to perform repeated blood draws via a vein in the leg, but were unsuccessful. Indeed, we did collect venous blood samples from the antecubital vein and analyzed the plasma for oxidative stress biomarkers. However, these biomarkers did not change during $3 \mathrm{hr}$ of sitting as compared to baseline similar to the brachial artery FMD implying the local lower extremity effect could not be detected using the upper extremity measurements(38). Finally, during the measurement of FMD, most of our subjects did not have any dilation of the SFA at least at one measurement point. Only 4 subjects had non-zero FMD\%. It is hence difficult to calculate and interpret SRauc which is considered to be the stimulus for the dilation, and interpret the FMD normalized to SRauc. It is possible that the change in the FDM\% was a direct result of the change in SRauc. However, based on the available data we cannot speculate this as a possible mechanism.

Despite these limitations, our study adds significant new information to the field of inactivity physiology. This is the first experimental evidence of the effects of prolonged sitting on human vasculature, performed using a robust protocol with all measurements taken in the seated position. 


\section{Conclusions}

SFA FMD exhibits an acute decline during $3 \mathrm{hr}$ of sitting. Starting at $1 \mathrm{hr}$, FMD in the SFA attenuates and remains significantly low through $3 \mathrm{hr}$ of quiet sitting. This is complimentary to the significant decline in mean and antegrade shear rates. The decline in FMD is counteracted by adding 5 min of light intensity physical activity $\left(2\right.$ miles.hr $\left.{ }^{-1}\right)$ bouts each hour during the sitting time. Three hours of sitting is a common phenomenon in different settings such as workplace, transportation, and leisure settings. Our results provide with first experimental evidence demonstrating that breaking sitting time protects endothelial function and hence may be anti-atherosclerotic in nature. We believe our observations further the argument to have structured public health guidelines on limiting sitting time.

\section{Acknowledgements}

The author thanks Samantha Mayhew and Chad Wiggins for their assistance during data collection.

Sources of Funding: This project was funded in part through American College of Sports Medicine Foundation's Doctoral Student Research Grant, and IU School of Public Health and IU University Graduate School's grant in aid awarded to SST.

Conflict of interest: The author reports no conflict of interest.

The results do not constitute endorsement by ACSM. 


\section{References}

1. Bauman AE, Chau JY, Ding D, Bennie J. Too Much Sitting and Cardio-Metabolic Risk: An Update of Epidemiological Evidence. Current Cardiovascular Risk Reports. 2013 (7):1-6.

2. CDC. How much physical activity do adults need? Downloaded from: http://www.cdc.gov/physicalactivity/everyone/guidelines/adults.html. Retrieved: December 8, 2013.

3. Davignon J, Ganz P. Role of endothelial dysfunction in atherosclerosis. Circulation. 2004;109(Supplement 231):III-27-III-32.

4. Dunstan DW, Kingwell BA, Larsen R et al. Breaking Up Prolonged Sitting Reduces Postprandial Glucose and Insulin Responses. Diabetes Care. 2012; 35(5):976-983.

5. Duvivier BM, Schaper NC, Bremers MA et al. Minimal intensity physical activity (standing and walking) of longer duration improves insulin action and plasma lipids more than shorter periods of moderate to vigorous exercise (cycling) in sedentary subjects when energy expenditure is comparable. PloS one. 2013;8(2):e55542.

6. Frydenlund G, Jørgensen T, Toft U, Pisinger C, Aadahl M. Sedentary leisure time behavior, snacking habits and cardiovascular biomarkers: the Inter99 Study. European Journal of Preventive Cardiology. 2012;19(5):1111-9.

7. Giannotti G, Landmesser U. Endothelial dysfunction as an early sign of atherosclerosis. Herz Kardiovaskuläre Erkrankungen. 2007;32(7):568-72. 
8. Harrington DM, Barreira TV, Staiano AE, Katzmarzyk PT. The descriptive epidemiology of sitting among US adults, NHANES 2009/2010. Journal of Science and Medicine in Sport. 2014;17(4):371-5.

9. Healy GN, Dunstan DW, Salmon J et al. Breaks in sedentary time. Diabetes Care. 2008;31(4):661-666.

10. Hijmering ML, Stroes ES, Olijhoek J, Hutten BA, Blankestijn PJ, Rabelink TJ. Sympathetic activation markedly reduces endothelium-dependent, flow-mediated vasodilation. Journal of the American College of Cardiology. 2002;39(4):683-8.

11. Hitosugi M, Niwa M, Takatsu A. Rheologic changes in venous blood during prolonged sitting. Thrombosis research. 2000;100(5):409-12.

12. Johnson BD, Mather KJ, Newcomer SC, Mickleborough TD, Wallace JP. Vitamin C prevents the acute decline of flow-mediated dilation after altered shear rate patterns. Applied Physiology, Nutrition, and Metabolism. 2012;38(3):268-74.

13. Johnson DG. Muscle sympathetic nerve activity during postural change in healthy young and older adults. Clinical Autonomic Research. 1995;5(1):57-60.

14. Kizhakekuttu TJ, Gutterman DD, Phillips SA et al. Measuring FMD in the brachial artery: how important is QRS gating? Journal of Applied Physiology. 2010;109(4):95965.

15. Kooijman M, Thijssen D, De Groot P et al. Flow-mediated dilatation in the superficial femoral artery is nitric oxide mediated in humans. The Journal of physiology. 2008;586(4):1137-45. 
16. Kröger K, Kucharczik A, Hirche H, Rudofsky G. Atherosclerotic lesions are more frequent in femoral arteries than in carotid arteries independent of increasing number of risk factors. Angiology. 1999;50(8):649-54.

17. Ku DN. Blood flow in arteries. Annual Review of Fluid Mechanics. 1997;29(1):399-434.

18. Kwaan HC. Role of plasma proteins in whole blood viscosity: a brief clinical review. Clinical hemorheology and microcirculation. 2010;44(3):167-76.

19. Malek AM, Alper SL, Izumo S. Hemodynamic shear stress and its role in atherosclerosis. JAMA: the journal of the American Medical Association. 1999;282(21):2035-42.

20. Newcomer SC, Sauder CL, Kuipers NT, Laughlin MH, Ray C. Effects of posture on shear rates in human brachial and superficial femoral arteries. American Journal of Physiology-Heart and Circulatory Physiology. 2008;294(4):H1833-H9.

21. Padilla J, Johnson BD, Newcomer SC et al. Normalization of flow-mediated dilation to shear stress area under the curve eliminates the impact of variable hyperemic stimulus. Cardiovasc Ultrasound. 2008;6(44):42.

22. Padilla J, Sheldon RD, Sitar DM, Newcomer SC. Impact of acute exposure to increased hydrostatic pressure and reduced shear rate on conduit artery endothelial function: a limbspecific response. American Journal of Physiology-Heart and Circulatory Physiology. 2009;297(3):H1103-H1108.

23. Padilla J, Young CN, Simmons GH et al. Increased muscle sympathetic nerve activity acutely alters conduit artery shear rate patterns. American Journal of Physiology-Heart and Circulatory Physiology. 2010;298(4):H1128-H35.

24. Pekarski SE. A gravitational hypothesis of essential hypertension as a natural adaptation to increased gravitational stress caused by regular, prolonged sitting typical of modern 
life. Medical science monitor: international medical journal of experimental and clinical research. 2004;10(6):HY27-HY32.

25. Pereira SMP, Ki M, Power C. Sedentary behaviour and biomarkers for cardiovascular disease and diabetes in mid-life: the role of television-viewing and sitting at work. PloS one. 2012;7(2):e31132.

26. Pyke KE. Should we be on the fence or can we open the gate? Evidence that QRS gating in FMD analysis is not essential. Journal of Applied Physiology. 2010;109(4):945-6.

27. Quyyumi AA, Patel RS. Endothelial Dysfunction and Hypertension Cause or Effect? Hypertension. 2010;55(5):1092-4.

28. Ross R, Wight T, Strandness E, Thiele B. Human atherosclerosis. I. Cell constitution and characteristics of advanced lesions of the superficial femoral artery. The American journal of pathology. 1984;114(1):79-93.

29. Saito M, Tsukanaka A, Yanagihara D, Mano T. Muscle sympathetic nerve responses to graded leg cycling. Journal of Applied physiology. 1993;75(2):663-7.

30. Shvartz E, Gaume J, White R, Reibold R. Hemodynamic responses during prolonged sitting. Journal of Applied Physiology. 1983;54(6):1673-80.

31. Stary HC, Chandler AB, Dinsmore RE et al. A definition of advanced types of atherosclerotic lesions and a histological classification of atherosclerosis A report from the Committee on Vascular Lesions of the Council on Arteriosclerosis, American Heart Association. Arteriosclerosis, thrombosis, and vascular biology. 1995;15(9):1512-31.

32. Stephens BR, Granados K, Zderic TW, Hamilton MT, Braun B. Effects of 1 day of inactivity on insulin action in healthy men and women: interaction with energy intake. Metabolism. 2011;60(7):941-9. 
33. Thijssen D, Dawson EA, Black MA, Hopman M, Cable NT, Green DJ. Brachial artery blood flow responses to different modalities of lower limb exercise. Med Sci Sports Exerc. 2009;41(5):1072-9.

34. Thijssen DHJ, Black MA, Pyke KE et al. Assessment of flow-mediated dilation in humans: a methodological and physiological guideline. American Journal of PhysiologyHeart and Circulatory Physiology. 2011;300(1):H2-H12.

35. Thosar S, Johnson BD, Johnston JD, Wallace JP. Sitting and endothelial function: the role of shear stress. Medical Science Monitor. 2012; 18(12): RA173-RA180.

36. Tinken TM, Thijssen DH, Hopkins $\mathrm{N}$ et al. Impact of shear rate modulation on vascular function in humans. Hypertension. 2009;54(2):278-85.

37. Vita JA, Keaney Jr JF. Endothelial Function A Barometer for Cardiovascular Risk? Circulation. 2002;106(6):640-2.

38. Wallace J. Does brachial artery endothelial function represent the endothelial function during prolonged sitting? (Abstract) Medicine \& Science in Sports \& Exercise. 2014;46(5 Suppl):S11-4.

39. Weiner SD, Jin Z, Cushman M et al. Brachial artery endothelial function and coagulation factors in the multi-ethnic study of atherosclerosis (MESA). Journal of the American College of Cardiology. 2011;57(14s1):E1414-E1414.

40. Young CN, Deo SH, Padilla J, Laughlin MH, Fadel PJ. Pro-atherogenic shear rate patterns in the femoral artery of healthy older adults. Atherosclerosis. 2010;211(2):390392. 


\section{Figure Legends}

Figure 1: Time line of study design. SIT refers to 3 hours of prolonged sitting. ACT refers to the breaking sitting time trial. $\mathcal{K}$ indicates walking on the treadmill for 5 minutes at 2 miles $^{-1} \mathrm{~h}^{-1}$ Walking commenced at $30 \mathrm{~min}, 1 \mathrm{hr} 30 \mathrm{~min}$ and $2 \mathrm{hr} 30 \mathrm{~min}$.

Figure 2: Flow mediated dilation in the superficial femoral artery during 3 hours of sitting during the sitting (SIT) and breaking sitting time (ACT trial). Error bars represent standard error. \# indicates significant difference from baseline in the SIT trial, * indicates significant difference between trials at $\mathrm{p} \leq 0.05$. 
Figure 1

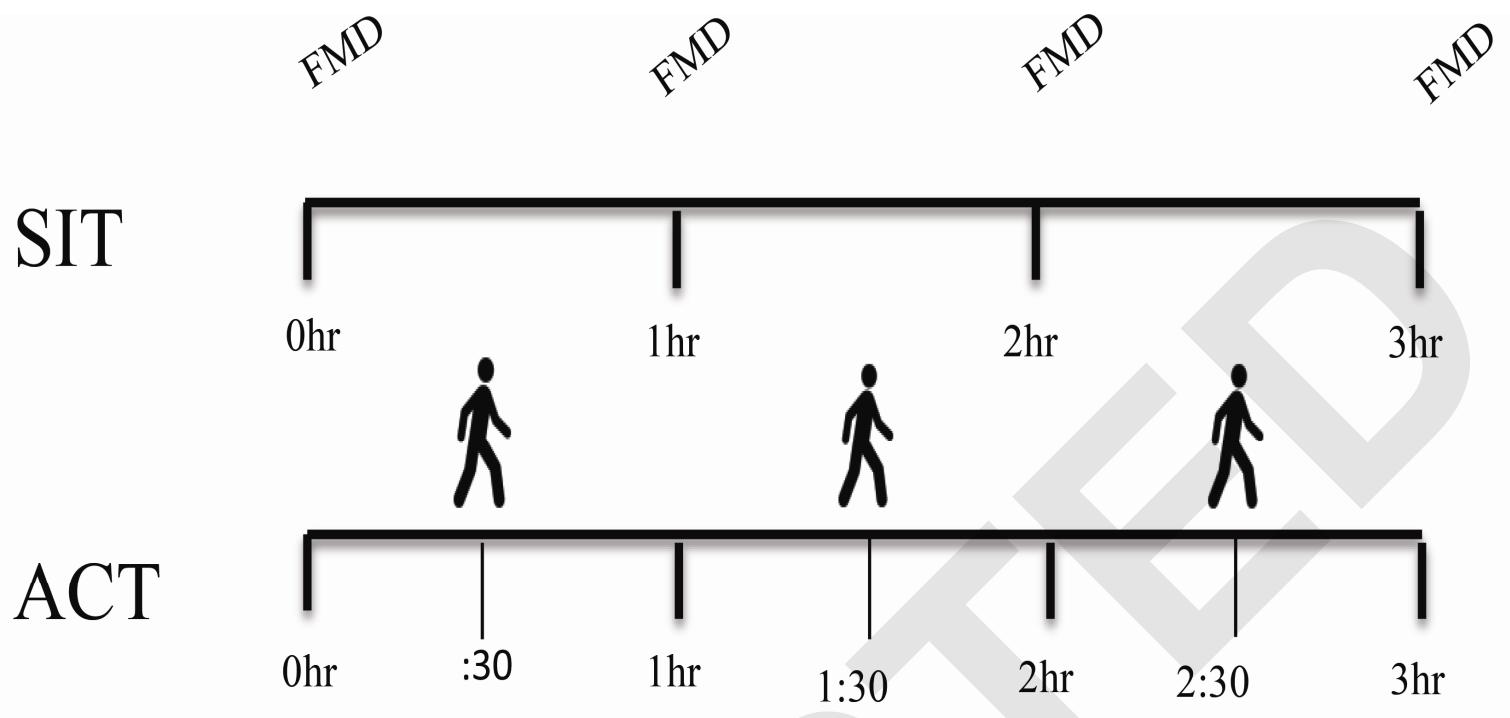


Figure 2

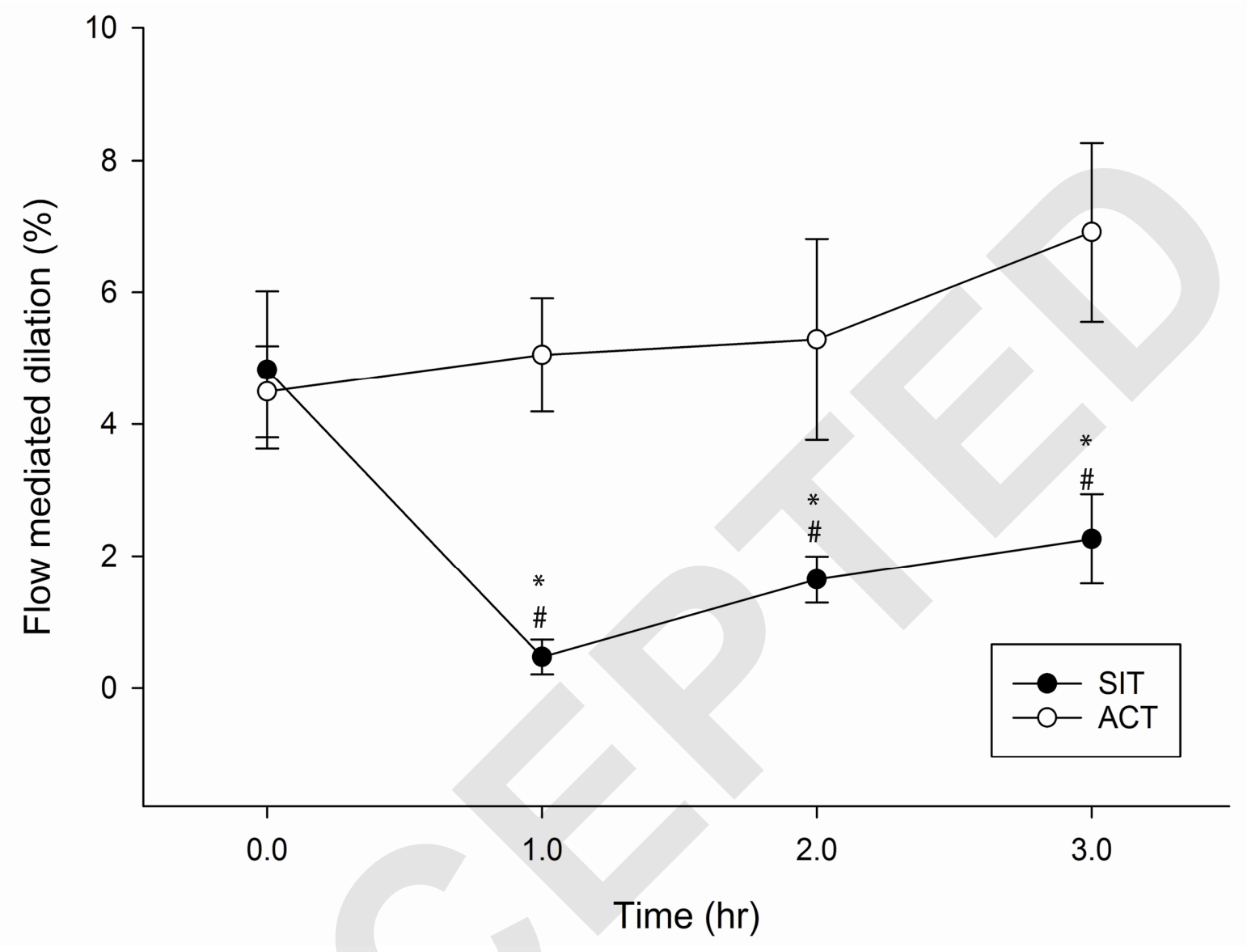


Table 1: Subject demographics

\begin{tabular}{ll}
\hline Variable & Value \\
\hline \hline $\mathrm{N}$ & 12 \\
Age $(\mathrm{yrs})$. & $24.2 \pm 4$ \\
BMI $\left(\mathrm{kg} . \mathrm{m}^{2}\right)$ & $23.7 \pm 3.4$ \\
Systolic blood pressure $(\mathrm{mm} \mathrm{Hg})$ & $115.9 \pm 9$ \\
Diastolic blood pressure (mm Hg) & $77.3 \pm 6.2$ \\
Total cholesterol (mg/dl) & $171 \pm 31.2$ \\
LDL cholesterol (mg/dl) & $98.17 \pm 28.2$ \\
HDL cholesterol (mg/dl) & $57.2 \pm 13$ \\
VLDL cholesterol (mg/dl) & $15.7 \pm 9$ \\
Triglycerides $(\mathrm{mg} / \mathrm{dl})$ & $77.67 \pm 47$ \\
Glucose $(\mathrm{mg} / \mathrm{dl})$ & $90.9 \pm 6$ \\
\hline
\end{tabular}

Data are presented as mean \pm SD 
Table 2: Baseline diameters (mm), Shear rates $\left(\mathrm{s}^{-1}\right)$, SRauc and normalized FMD in the SFA during the SIT and ACT trial at baseline, $1 \mathrm{hr}, 2 \mathrm{hr}$ and $3 \mathrm{hr}$.

\begin{tabular}{|c|c|c|c|c|c|c|c|c|}
\hline & & & SIT & & & & $\mathrm{ACT}$ & \\
\hline & Ohr & $1 \mathrm{hr}$ & $2 \mathrm{hr}$ & $3 \mathrm{hr}$ & $\mathrm{Ohr}$ & $1 \mathrm{hr}$ & $2 \mathrm{hr}$ & $3 \mathrm{hr}$ \\
\hline $\begin{array}{l}\text { Baseline } \\
\text { diameters }\end{array}$ & $6.32 \pm 0.63$ & $6.37 \pm 0.71$ & $6.52 \pm 0.68$ & $6.32 \pm 0.74$ & $6.50 \pm 0.21$ & $6.40 \pm 0.17$ & $6.31 \pm 0.19$ & $6.22 \pm 0.21$ \\
\hline Antegrade & $7.14 \pm 0.69$ & $5.26 \pm 0.79^{\wedge}$ & $5.66 \pm 0.72^{\wedge}$ & $5.89 \pm 0.55$ & $5.87 \pm 0.83$ & $8.34 \pm 2.92$ & $5.45 \pm 0.71$ & $5.61 \pm 0.85$ \\
\hline Mean & $4.53 \pm 0.66$ & $2.42 \pm 0.96^{\wedge}$ & $2.81 \pm 0.50^{\wedge}$ & $3.38 \pm 0.47$ & $3.64 \pm 0.62$ & $5.32 \pm 2.90$ & $2.94 \pm 0.69$ & $2.69 \pm 0.60$ \\
\hline Retrograde & $2.62 \pm 0.36$ & $2.83 \pm 0.54$ & $2.85 \pm 0.45$ & $2.51 \pm 0.32$ & $2.23 \pm 0.40$ & $3.02 \pm 0.55$ & $2.51 \pm 0.44$ & $2.92 \pm 0.43$ \\
\hline Peak & $52.04 \pm 21.20$ & $19.21 \pm 4.27$ & $27.38 \pm 4.41$ & $23.08 \pm 3.11$ & $22.14 \pm 8.67$ & $21.44 \pm 3.23$ & $18.85 \pm 2.86$ & $19.01 \pm 2.04$ \\
\hline SRauc(a.u) & $838.69 \pm 134.27$ & $442.63 \pm 178.73$ & $363.83 \pm 127.30$ & $580.18 \pm 136.42$ & $296.35 \pm 132.72$ & $321.82 \pm 134.60$ & $299.53 \pm 127.25$ & $477.39 \pm 106.68$ \\
\hline FMD:SRauc & $.0067 \pm .0035$ & $.008 \pm .0089$ & $.0041 \pm .0034$ & $.0041 \pm .0038^{*}$ & $.031 \pm .0296$ & $.0203 \pm .0190$ & $.0195 \pm .0133$ & $.0157 \pm .0075^{*}$ \\
\hline
\end{tabular}

SFA shear rates $\left(\mathrm{s}^{-1}\right)$ and baseline diameters $(\mathrm{mm})$ during SIT and ACT trials. Data represented as Mean \pm SE

$\wedge$ Indicates significantly different from $0 \mathrm{hr}$ in SIT.

*Indicates significantly different from each other

Note: Data for SRauc and FMD: SRauc are from 4 participants. See limitations for details. 